\title{
Central nervous system SIRT1 expression is required for cued and contextual fear conditioning memory responses in aging mice
}

\author{
Devin Wahl $^{\mathrm{a}, \mathrm{b}, 1, *}$, Rahul Gokarn ${ }^{\mathrm{a}, 1}$, Sarah J. Mitchell ${ }^{\mathrm{c}}$, Samantha M. Solon-Biet ${ }^{\mathrm{a}}$, \\ Victoria C. Cogger ${ }^{\mathrm{a}, \mathrm{b}}$, Stephen J. Simpson ${ }^{\mathrm{a}, \mathrm{d}}$, David G. Le Couteur ${ }^{\mathrm{a}, \mathrm{b}}$ and Rafael de Cabo \\ ${ }^{a}$ Charles Perkins Centre, University of Sydney, Sydney, Australia \\ ${ }^{\mathrm{b}}$ Aging and Alzheimer's Institute, ANZAC Research Institute, Concord Clinical School/Sydney \\ Medical School, Concord, Australia \\ ${ }^{\mathrm{c}}$ Harvard T.H. Chan School of Public Health, Boston, MA, USA \\ ${ }^{\mathrm{d}}$ School of Life and Environmental Sciences, University of Sydney, Sydney, Australia \\ ${ }^{\mathrm{e}}$ Translational Gerontology Branch, National Institute on Aging, National Institutes of Health, \\ Baltimore, MD, USA
}

\begin{abstract}
.
BACKGROUND: Sirtuin 1 (SIRT1) is a NAD ${ }^{+}$-dependent enzyme that has important roles in many biological processes involved in aging, including cell growth and repair, inflammation, and energy regulation. SIRT1 activity is modulated in response to certain nutritional interventions that increase healthspan and longevity in rodents, including calorie restriction (CR) and intermittent fasting (IF). In addition to positively influencing cardiometabolic health, SIRT1 is important for brain health and may be critical in the preservation of memory processes that deteriorate during aging.

OBJECTIVE: To investigate the role of brain-associated SIRT1 expression in the acquisition of fear memory in mice at 45 and 65 weeks of age.

METHODS: Mice with brain-specific knock-out or overexpression of Sirtl were assessed on a fear conditioning paradigm to determine the role of SIRT1 in fear memory acquisition.

RESULTS: In the current study, mice lacking the expression of brain SIRT1 could not learn the fear conditioning paradigm during training, context, or cue phases.

CONCLUSIONS: The results of the study indicate that SIRT1 expression in the brain is critical for the formation of fear memory in male mice at two distinct ages, highlighting the essential role of SIRT1 in fear memory acquisition during aging.
\end{abstract}

Key words: SIRT1, Fear conditioning, classically conditioned memory, aging, hippocampus

\section{Introduction}

Mammalian sirtuins are a conserved family of seven $\mathrm{NAD}^{+}$-dependent deacetylases that regulate

\footnotetext{
${ }^{1}$ These authors contributed equally to this work.

*Corresponding author: Devin Wahl, PhD, Charles Perkins Centre, University of Sydney, Sydney, Australia. Tel.: +614029 01344; E-mail: devin.wahl@ sydney.edu.au.
}

many biological aging processes, including cell growth and repair, inflammation, development, and energy regulation $[1,2]$. Sirtuin 1 (SIRT1) has important roles in neurobiological processes that include cognitive and behavioural responses during aging including classical conditioning memory [3]. It is well known that SIRT1 activity can modulated by nutritional interventions that improve healthspan and 
lifespan, including calorie restriction (CR) and intermittent fasting (IF) [4]. In addition to those nutritional interventions, it has been suggested that pharmacologically increasing brain SIRT1 expression may provide a promising avenue for the hinderance and treatment of neurodegenerative disease including Alzheimer's disease (AD) and related dementias [5].

SIRT1 expression in the nervous system is essential for hippocampus-dependent and independent memory processes during aging [6]. As aging is the greatest risk factor for the development of neurodegeneration, SIRT1 may be a plausible target to prevent the onset of neurodegenerative disease [7, 8]. Moreover, SIRT1 activity in the brain declines with age [9] possibly contributing to a reduction in hippocampal synaptic plasticity and memory function. There have been recent studies $[10,11]$ that have investigated the role of central nervous system SIRT1 expression in behavioral-memory responses in mice, but few have looked at the effect of SIRT1 expression on memory in mice of different ages. Therefore, the current study builds upon the previous literature by assessing the role of central nervous system SIRT1 expression in a classically conditioned fear memory paradigm in mice of two distinct ages, thereby providing insights into the role of brain specific SIRT1 expression on memory responses in aging mice.

In humans, the gradual loss of contextual memory is a main hallmark of the aging brain, hippocampus degeneration, and the onset of neurodegenerative disease $[12,13]$. Therefore, the purpose of the current study was to examine the role of SIRT1 expression in contextual and cued memory responses in male C57/B6 mice. Mice with either brain-specific knockout (KO) or overexpression (OE) of Sirtl versus age-matched littermate controls (LM) were assessed on the fear conditioning behavioural paradigms to determine the role of centrally expressed SIRT1 in classically conditioned fear memory in 45 weeks old and 65 weeks old male mice.

\section{Materials and methods}

\subsection{Animal husbandry and diets}

Mice with brain-specific knockout (KO) and overexpression (OE) of Sirtl were generated using the Nestin-Cre system on C57BL/6 background as previously described [3]. Mice were housed 3-4 per cage with ad-libitum access to water at the National Institute on Aging (Baltimore, MD, USA). Body weight and food consumption measurements were taken bi-weekly and mice were routinely checked for health status. Mice were fed house chow (2018 Teklad Global 18\% protein rodent diet, 2018S, Envigo, Somerset NJ) with all necessary vitamins and minerals for optimal rodent growth and development. Measures of lean, fat, and fluid mass were acquired in live mice by NMR (Minispec LF90, Bruker Optics, Billerica, MA, USA). This study was approved by the National Institute on Aging, animals care and use committee (352-TGB-2015). All mice were treated and cared for in accordance with the guidelines of the National Institute on Aging (NIA).

\subsection{Fear conditioning}

Male brain-specific KO, brain-specific OE and littermate (LM) control mice at 45 and 65 weeks of age underwent fear conditioning. Before testing, mice were habituated to the testing room for a minimum of 2 hours. For training, individual mice were placed in a sound-proof contextual conditioning chamber (Med Associates, St Albans, VT, USA) containing a metal grid on the floor. At the end of a 2-min exploration time, mice were subjected to three sessions of audio tone (conditioned stimulus) directly followed by a mild foot shock (unconditioned stimulus). The audio tone $(5 \mathrm{kHz}, 70 \mathrm{~dB})$ was played for $30 \mathrm{~s}$ followed by a $0.5 \mathrm{~mA}$ foot shock for $2 \mathrm{~s}$ from the metal grid floor. Thirty seconds separated each tone and shock in the training session. Mice were returned to the same conditioning chamber $24 \mathrm{~h}$ after the initial training session for $5 \mathrm{~min}$, but without any shock or audio tone. The percentage of freezing time was recorded by the software and used as an index of contextual memory. The cued test was performed $2 \mathrm{~h}$ after the contextual test. First, a non-toxic chocolate fragrance was applied lightly to the bedding to change the smell of the chamber, and black and white striped pictures were placed on the back and side chamber walls to change the chamber appearance. Mice were let to explore for $5 \mathrm{~min}$ without any audio tone, followed by three tones (without a foot shock) that were played for $30 \mathrm{~s}$ each. The percentage of freezing time during and after the audio tones was recorded by the software and used as a measure of cued memory response. Data are presented as the percentage of freezing time during each test. 

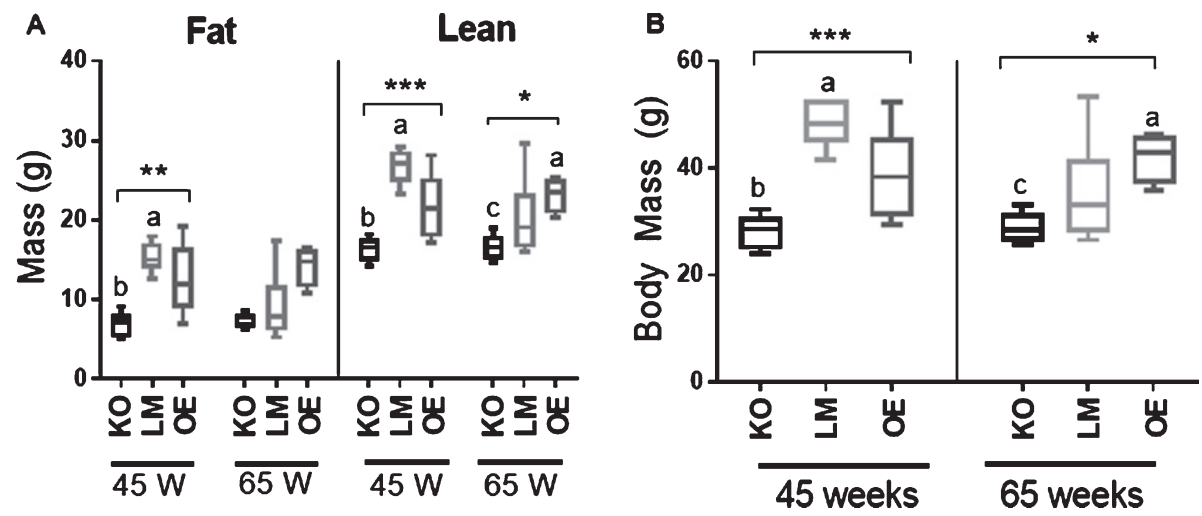

Fig. 1. Body composition analysis in male mice. (A) Fat mass and lean mass in mice with brain-specific knockout (KO) or overexpression (OE) of SIRT1 vs. littermate controls (LM) at 45 and 65 weeks of age. $n=4-10$ mice per experimental group. (B) Body weight measurement; $n=4$-10 mice per experimental group. ${ }^{*} p<0.05,{ }^{* *} p<0.01,{ }^{* * *} p<0.001,{ }^{* * * *} p<0.0001$. Error bars represent the mean \pm SEM. Different letters $(\mathrm{a}, \mathrm{b}, \mathrm{c})$ indicate statistically significant differences as determined from the Dunn's post-hoc test corrected for multiple comparisons. $a=$ group is significantly different to $\mathrm{KO} ; b=$ group is significantly different to LM; $c=$ group is significantly different to OE.

\subsection{Statistical analysis}

First, a D'Agostino and Pearson test was performed to determine whether the data were normally distributed. To account for the uneven group sizes, a nonparametric Kruskal-Wallis test - one-way analysis of variance (ANOVA) coupled with a Dunn's post-hoc test with a correction for multiple comparisons was to determine the differences among the groups. Data are represented as means \pm SEM and $p$ values of less than 0.05 were considered statistically significant.

\section{Results}

\subsection{SIRT1 brain-specific KO mice exhibit changes in fat mass, lean mass, and body weight}

It has been well-established that SIRT1 plays a critical role in mitochondrial function and energy expenditure [14]. Therefore, we investigated whether the mice in the current study exhibited changes in total body weights or body composition. When compared to age-matched SIRT brain-specific OE and LM mice, SIRT1 brain-specific KO animals at 45 weeks of age exhibited a significantly lower fat mass $(n=5$ 8 mice per group, $p<0.01$ ), while the 65 -week-old SIRT1 brain specific KO mice had a nearly significant $(p=0.0513)$ reduction in fat mass $(n=4-10$ mice per group). Similarly, a significant reduction in lean mass (Fig. 1A) and body weight (Fig. 1B) was observed in SIRT1 brain-specific KO mice of both ages when compared to the age-matched SIRT brain-specific OE and LM controls.

\subsection{SIRT1 is required for normal fear conditioning response in male mice}

In addition to affecting cardiometabolic health, SIRT1 may also be critical for memory responses in aging mice [7]. Therefore, a well-established fear conditioning paradigm was carried out to determine if brain-specific SIRT1 influenced classically conditioned fear memory. A fear conditioning test measured the acquisition of contextual and cued memory in an adverse classical conditioning learning paradigm. There were significant differences among the groups during the training phase where the SIRT1 brain-specific $\mathrm{KO}$ mice of both ages were unable to learn to associate the tone with an adverse outcome during the three stimulus parings during training on day 1 (Fig. 2) SIRT1 brain-specific KO mice at 45 (Fig. 2A) and 65 weeks (Fig. 2B) of age could not learn to associate the same environment with a foot shock (contextual) nor the tone with a foot shock (cued) during either testing phase. The lack of differences in fear conditioned memory between brain-specific OE and LM mice of both ages suggests that SIRT1 overexpression does not improve contextual or cued fear conditioning memory in male mice. 

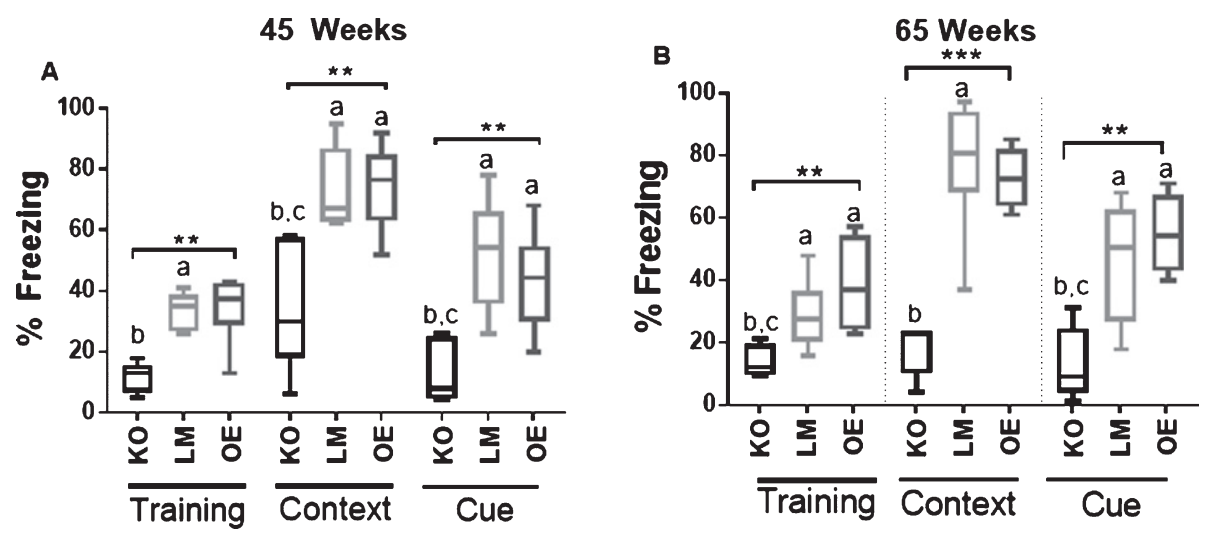

Fig. 2. Fear conditioning responses in male mice. Data are presented as the percentage of freezing time during testing. Fear conditioning responses in (A) 45-week-old and (B) 65-week-old mice. $n=5-8$ mice (A) and 4-10 mice (B) per experimental group. KO = brain-specific SIRT1 knock-out, OE = brain-specific SIRT1 overexpression, LM= littermate controls. ${ }^{* *} p<0.01,{ }^{* * *} p<0.001$. Error bars represent the mean \pm SEM. Different letters (a,b,c) indicate statistically significant differences as determined from the Dunn's post-hoc test corrected for multiple comparisons. $a=$ group is significantly different to $\mathrm{KO} ; b=$ group is significantly different to LM; $c=$ group is significantly different to OE.

\section{Discussion}

The link between SIRT1 expression and activity with brain health has been extensively investigated. SIRT1 is important for maintaining neuronal mitochondrial respiration and to reduce apoptosis [15], attenuate inflammation [16], preserve axons, and improve neurite outgrowth during aging [17]. SIRT1 also contributes to the maintenance of memory processes that deteriorate during normal aging [18]. For example, the deacetylation of SIRT1 target proteins and the modulation of several pathways, including SIRT1-p53 and SIRT1-AMPK, are known to influence memory capacity measured using cognitive and behavioural tasks in mice [19]. Additionally, it is well known that SIRT1 activity is increased with dietary patterns that improve cardiometabolic health and delay the onset of neurodegenerative disease in rodents. Some of those common dietary patterns include CR, IF, and low-protein, high carbohydrate (LPHC) diets [20]. The results of the current study provide additional support to suggest that adherence to these diets may be protective against associative memory loss during aging, partly due to increased brain SIRT1 activity and expression levels.

There have been a number of cognitive-behavioral studies that investigated the role of central nervous system SIRT1 in memory response in mice but most assessed memory responses at only one age. Gao and colleagues assessed tone and contextual fear conditioning memory responses in $5-6$ months old SIRT1 knockout mice. Comparable to our study, the authors found that the mutant mice lacking brain SIRT1 exhibited a significant decrease in freezing behaviour when compared to LM controls [10]. Similarly, Michán and colleagues assessed the role of SIRT1 expression in fear conditioning response in mice aged 5-10 months and again demonstrated a similar result to the current study [3]. The purpose of this study, therefore, was twofold: (1) Confirming the results of those previous studies which demonstrated the importance of brain specific SIRT1 expression in classically conditioned fear memory in mice; and (2) Including a novel dimension by testing mice at two developmental stages to provide insight into the role of central nervous system expression during aging. The result here provides continued evidence for the importance of central nervous system SIRT1 expression in classically conditioned memory responses during aging [9].

One possible explanation for how SIRT1 confers neuroprotection is its role in the regulation of energy metabolism and mitochondrial function during aging. Mitochondrial dysfunction increases with age which, in the brain, partly contributes to memory impairment [21]. It has been established that abnormal energy metabolism may be associated with adverse health outcomes, including neurodegenerative disease, during aging. SIRT1 has been implicated in glucose and lipid metabolism and energy expenditure, partly by influencing hypothalamic pathways [14]. Therefore, in the current study, we investigated whether brain-specific SIRT1 influenced body weights and lean and fat mass. In the current study, the SIRT1 
brain-specific KO mice exhibited reduced fat mass, lean mass, and body weight when compared to the other groups of animals. There have been several studies that assessed several metabolic parameters in SIRT1-null mice with ad-libitum access to food. The SIRT1-null mice weighed less and had lower activity measures and respiratory exchange ratios than their littermate counterparts [22]. Our study suggests that brain-specific SIRT1 expression may be key for the maintenance of gross body weight and body composition during aging.

The hippocampus is one of the first regions of the rodent and human brain that deteriorates with aging and neurodegenerative diseases [23]. Fear conditioning is a well-established cognitive-behavioural test to examine memory during aging. There is emerging evidence to suggest that fear memory is mediated by several brain areas, including the prefrontal cortex [24]. A well-established consensus, however, is that fear memory relies heavily on the amygdala and hippocampus, both of which lie in the limbic system. There is emerging evidence to suggest that both the hippocampus and the amygdala are important for both contextual and cued memory [25]. Additionally, the amygdala and the hippocampus form an intricate connection and both are highly involved in the formation of emotional memory. Therefore, damage or reduced function associated with either region may contribute to the early decline of emotional memory, which is often an early symptom of neurodegenerative disease including dementia [26].

While we did not directly assess any neurophysiological or biochemical parameters in current investigation, there have been a number of recent studies that looked at the roles of brain-specific SIRT1 on physiological and biochemical processes in the rodent hippocampus and amygdala. One study showed that SIRT1 overexpression in the hippocampus was sufficient to protect against Alzheimer's disease ( $\mathrm{A} \beta$ and $\mathrm{Tau}$ ) pathologies and improve spatial and recognition memory in triple transgenic mice. The authors hypothesised that the effects were largely due to an increase in brain-derived neurotropic factors and neuronal plasticity, and a decrease in proteostatic dysfunction [8]. Additional studies have revealed that SIRT1 overexpression in the brain has a direct impact on anxiety and behavioural responses in mice. It was hypothesised that this result may have partly been due to changes in the concentrations of key neurotransmitters involved in memory and cognition, including Serotonin and its main metabolite, 5 hydroxyindoleacetic acid [27]. Importantly, optimal
SIRT1 expression is necessary for optimal brain health during aging and has been promoted in axon and dendrite growth and branching [17].

The results of this study highlight the importance of brain-associated SIRT1 expression in hippocampusdependent and hippocampus-independent forms of fear-conditioning memory in mice. We found that the SIRT1 brain-specific KO mice were unable to learn to associate the same environment, nor tone, with an adverse outcome. Moreover, these mice were incapable to learn to associate the tone with an adverse event during the three stimulus pairings during training, which further highlights the importance of SIRT1 in the first stages of memory acquisition. There were no significant differences between the SIRT1 brain-specific OE and LM mice of both ages, which suggests that the overexpression of SIRT1 in the brain does not improve memory.

Aging is the single greatest contributor to the development of neurodegenerative disorders, including Alzheimer's disease [28]. The results of the current study may have important implications for aging research because it has been demonstrated that SIRT1 levels in the brain decline during aging at both transcriptional and translational levels [29]. Therefore, increasing SIRT1 expression through dietary or pharmacological means may be an effective way to reduce the burden of neurodegenerative disease in humans. One such example of a molecule that may be used for this purpose is resveratrol (RSV), which is an allosteric activator of SIRT1 [30]. In addition to reducing the major hallmarks of brain aging in rodents, [31] there is now ample evidence that RSV supplementation may also be effective to help protect against neurodegenerative disease and memory loss in humans, largely through the activation of SIRT1 [32].

There are several limitations to the current study. Firstly, there was only a five-month age gap between the two cohorts of mice (45 vs 65 weeks-old). Nonetheless, the study provides insight into how differential SIRT1 expression in the brain affects memory at two time points and thus highlights the importance of SIRT1 during aging in a validated memory test. Secondly, only male mice were used in the current study. Nevertheless, the results provide insight into the role of SIRT1 expression and fear conditioning memory responses in a commonly used rodent model. There is a consensus that male and female rodents respond differently (largely due to differences in sex hormones) to contextual behavioural testing [33] and, therefore, it is imperative that future 
work in the field also includes female mice. The observed cognitive changes in the 65-weeks-old mice may have been partly due to a deterioration in global cognitive function, rather than the effects of SIRT1. Nonetheless, the study was well-controlled by including separate groups of mice at distinct ages, again highlighting the important role of brain-specific SIRT1 in fear conditioned memory responses at two distinct ages. Finally, although there were low numbers of mice in several groups, the memory effects of knocking out brain-specific SIRT1 were so large that we detected significant changes among the groups, largely due to the increased effect size.

SIRT1 is required for normal fear conditioning memory in C57BL/6 male mice at two different ages, thus highlighting the critical role of brain-specific SIRT1 in classically conditioned memory responses during aging. SIRT1 KO mice could not learn the fear conditioning task, suggesting that brain-specific SIRT1 expression is crucially important for the acquisition of cued and contextual memory. Moreover, the overexpression of brain-specific SIRT1 did not result in a significant improvement of classically conditioned fear memory in the mice. Taken together, the results of the current study suggest that targeting SIRT1, either pharmacologically or through nutritional interventions, may be a promising avenue for the treatment or hinderance of neurodegenerative disease and related memory loss during aging.

\section{Acknowledgments}

We gratefully acknowledge the care and assistance of the Comparative Medicine Section of the National Institute on Aging and in particular Dawn Nines and Dawn Boyer. We also wish to thank Dr. Michel Bernier for his critical comments and careful editorial advice. This work was supported by the National Health and Medical Research Council (Project Grant \# 1084267), the Ageing and Alzheimer's Association, Sydney Medical School Foundation, the American Australian Association and by the Intramural Research Program of the National Institute on Aging, National Institutes of Health.

\section{Funding}

The authors report no funding.

\section{Declaration of interest}

None declared.

\section{References}

[1] Michan S, Sinclair D. Sirtuins in mammals: Insights into their biological function. Biochem J. 2007;404(1):1-13.

[2] Haigis MC, Sinclair DA. Mammalian sirtuins: Biological insights and disease relevance. Annu Rev Pathol. 2010;5: 253-95.

[3] Michan S, Li Y, Chou MM, Parrella E, Ge H, Long JM, et al. SIRT1 is essential for normal cognitive function and synaptic plasticity. J Neurosci. 2010;30(29):9695-707.

[4] Chang HC, Guarente L. SIRT1 and other sirtuins in metabolism. Trends in endocrinology and metabolism: TEM. 2014;25(3):138-45.

[5] Kim D, Nguyen MD, Dobbin MM, Fischer A, Sananbenesi F, Rodgers JT, et al. SIRT1 deacetylase protects against neurodegeneration in models for Alzheimer's disease and amyotrophic lateral sclerosis. Embo j. 2007;26(13):3169-79.

[6] Satoh A, Imai SI, Guarente L. The brain, sirtuins, and ageing. Nat Rev Neurosci. 2017;18(6):362-74.

[7] Jesko H, Wencel P, Strosznajder RP, Strosznajder JB. Sirtuins and Their Roles in Brain Aging and Neurodegenerative Disorders. Neurochemical research. 2017;42(3):876-90.

[8] Corpas R, Revilla S, Ursulet S, Castro-Freire M, Kaliman P, Petegnief V, et al. SIRT1 Overexpression in Mouse Hippocampus Induces Cognitive Enhancement Through Proteostatic and Neurotrophic Mechanisms. Molecular neurobiology. 2017;54(7):5604-19.

[9] Ng F, Wijaya L, Tang BL. SIRT1 in the brain-connections with aging-associated disorders and lifespan. Frontiers in cellular neuroscience. 2015;9:64.

[10] Gao J, Wang WY, Mao YW, Graff J, Guan JS, Pan L, et al. A novel pathway regulates memory and plasticity via SIRT1 and miR-134. Nature. 2010;466(7310):1105-9.

[11] Libert S, Pointer K, Bell EL, Das A, Cohen DE, Asara JM, et al. SIRT1 activates MAO-A in the brain to mediate anxiety and exploratory drive. Cell. 2011;147(7):1459-72.

[12] Goodarzi Z, Mele B, Guo S, Hanson H, Jette N, Patten S, et al. Guidelines for dementia or Parkinson's disease with depression or anxiety: A systematic review. BMC Neurol. 2016;16(1):244.

[13] El Haj M, Kessels RP. Context memory in Alzheimer's disease. Dement Geriatr Cogn Dis Extra. 2013;3(1):342-50.

[14] Li X. SIRT1 and energy metabolism. Acta biochimica et biophysica Sinica. 2013;45(1):51-60.

[15] Zhou Y, Wang S, Li Y, Yu S, Zhao Y. SIRT1/PGC-1alpha Signaling Promotes Mitochondrial Functional Recovery and Reduces Apoptosis after Intracerebral Hemorrhage in Rats. Front Mol Neurosci. 2017;10:443.

[16] Meng X, Tan J, Li M, Song S, Miao Y, Zhang Q. Sirt1: Role Under the Condition of Ischemia/Hypoxia. Cell Mol Neurobiol. 2017;37(1):17-28.

[17] Herskovits AZ, Guarente L. SIRT1 in neurodevelopment and brain senescence. Neuron. 2014;81(3):471-83.

[18] Wahl D, Cogger VC, Solon-Biet SM, Waern RV, Gokarn R, Pulpitel T, et al. Nutritional strategies to optimise cognitive function in the aging brain. Ageing Res Rev. 2016;31:80-92.

[19] Cao Y, Yan Z, Zhou T, Wang G. SIRT1 Regulates Cognitive Performance and Ability of Learning and Memory in Diabetic and Nondiabetic Models. J Diabetes Res. 2017;2017:7121827.

[20] Wahl D, Coogan SC, Solon-Biet SM, de Cabo R, Haran JB, Raubenheimer D, et al. Cognitive and behavioral eval- 
uation of nutritional interventions in rodent models of brain aging and dementia. Clinical interventions in aging. 2017;12: 1419-28.

[21] Ham PB, 3rd, Raju R. Mitochondrial function in hypoxic ischemic injury and influence of aging. Prog Neurobiol. 2017;157:92-116.

[22] Boily G, Seifert EL, Bevilacqua L, He XH, Sabourin G, Estey C, et al. SirT1 regulates energy metabolism and response to caloric restriction in mice. PLoS One. 2008;3(3):e1759.

[23] Bettio LEB, Rajendran L, Gil-Mohapel J. The effects of aging in the hippocampus and cognitive decline. Neurosci Biobehav Rev. 2017;79:66-86.

[24] Giustino TF, Maren S. The Role of the Medial Prefrontal Cortex in the Conditioning and Extinction of Fear. Front Behav Neurosci. 2015;9:298.

[25] Izquierdo I, Furini CR, Myskiw JC. Fear Memory. Physiol Rev. 2016;96(2):695-750.

[26] McDonald AJ, Mott DD. Functional neuroanatomy of amygdalohippocampal interconnections and their role in learning and memory. J Neurosci Res. 2017;95(3):797-820.

[27] Libert S, Pointer K, Bell EL, Das A, Cohen DE, Asara JM, et al. SIRT1 activates MAO-A in the brain to mediate anxiety and exploratory drive. Cell. 2011;147(7):1459-72.
[28] Partridge L, Deelen J, Slagboom PE. Facing up to the global challenges of ageing. Nature. 2018;561(7721):45-56.

[29] Gong H, Pang J, Han Y, Dai Y, Dai D, Cai J, et al. Age-dependent tissue expression patterns of Sirt1 in senescence-accelerated mice. Molecular medicine reports. 2014;10(6):3296-302.

[30] Wahl D, Bernier M, Simpson SJ, de Cabo R, Le Couteur DG. Future directions of resveratrol research. Nutrition and healthy aging. 2018;4(4):287-90.

[31] Cao W, Dou Y, Li A. Resveratrol Boosts Cognitive Function by Targeting SIRT1. Neurochemical research. 2018;43(9):1705-13.

[32] Sawda C, Moussa C, Turner RS. Resveratrol for Alzheimer's disease. Annals of the New York Academy of Sciences. 2017;1403(1):142-9.

[33] Duarte-Guterman P, Yagi S, Chow C, Galea LA. Hippocampal learning, memory, and neurogenesis: Effects of sex and estrogens across the lifespan in adults. Horm Behav. 2015;74:37-52. 